\title{
Atrial septal defect patients with greater shunts show susceptibility for ventricular arrhythmias
}

\author{
Osman Can Yontar \\ University of Health Sciences Turkey, Samsun Training and Research Hospital, Cardiology Clinic, Samsun, Turkey
}

\begin{abstract}
Objective: Ventricular arrhythmia episodes are not infrequent in patients with atrial septal defect (ASD). Disturbance in cardiac volume and pressures may lead to enlargement and fibrosis in heart. An interatrial volume displacement through septal defect, briefly interatrial shunt, is the major reason for this complication. Prolongation of the interval between the peak and end of the T wave (Tpeak to Tend, Tp-e) on the 12-lead electrocardiogram (ECG), is utilized as a marker of ventricular arrhythmogenesis during last years. The aim of this study was to assess if there is an impact of shunt ratio on ventricular repolarization in patients with ASD by using Tp-e interval, Tp-e/QT ratio, and Tp-e/QTc ratio.

Methods: Patient records of Samsun Training and Research Hospital were retrospectively analyzed. Electrocardiograms of 133 patients, who were diagnosed as ASD between January 2016 and December 2019 were obtained and scanned. ECG intervals were measured. Shunt ratios, right ventricle diameters and volumes were also acquired. Patients were grouped into two by their calculated shunt ratio, ratio of $\geq 2.0$ is accepted as a high shunt group and $<2.0$ as a low shunt group.

Results: Both groups' baseline characteristics were similar. Right ventricular dimensions and systolic pulmonary artery pressure were higher in high shunt group. Furthermore, ASD patients with higher shunt ratio had significantly higher ECG measurements than controls, Tp-e: 103.0 (22.1) vs 76.2 (10.2); Tp-e/QT: 0.25 (0.03) vs 0.21 (0.02); Tpe/QTc: 0.22 (0.03) vs, 0.17 (0.02); for all p<0.001). Of all ECG parameters; Tp-e $(r=0.631, p<0.001), T p-e / Q T(r=0.531$, $\mathrm{p}<0.001)$ and Tp-e/QTc $(r=0.614, p<0.001)$ had moderate correlation with shunt ratio.

Conclusion: $T$ wave peak-to-end interval is a measure of transmural dispersion of repolarization and accepted as a surrogate for increased ventricular arrhythmogenesis risk. Our findings show that ASD patients whose shunt ratio are $\geq 2.0$ show increased risk for arrhythmias.
\end{abstract}

Key words: atrial septal defect, electrocardiogram, ventricular arrhythmia, risk, ventricular repolarization

(Heart Vessels Transplant 2020; 4; doi: 10.24969/hvt.2020.206)

\section{Introduction}

Atrial septal defect (ASD) constitutes a significant amount of congenital heart diseases (1). Secundum ASD is the most common type of ASD that causes diversion of blood from systemic to pulmonary circulation. In early stages of life, most patients are asymptomatic. Although some patients are diagnosed early, many of them remain silent until adulthood (2). If the disease stays unnoticed, decrease in exercise capacity, miscellaneous supraventricular arrhythmias, right heart failure, and pulmonary arterial hypertension may occur within years $(3,4)$. ASD may lead to disruptions in cardiac kinetics dependent on the defect diameter and shunt volume. Shunts which are calculated to be over 2.0 indicate large ASDs with significant blood diversion from left to right atria (5). If atrial and ventricular myocardium is exposed to volume overload, shear stress extends to not only myofibers but also conduction system of heart. Disruptions may manifest as conduction delay within atrium and ventricles $(6,7)$. Furthermore, the degeneration of cardiac microarchitecture, including enhanced fibrosis, improperly modifies myocardial repolarization $(6,7)$.

Address for Correspondence: Osman Can Yontar, University of Health Sciences Turkey, Samsun Training and Research Hospital, Cardiology Clinic, Samsun, Turkey Email: drcanyontar@gmail.com

Received: 13.06.2020 Revised: 04.07.2020 Accepted: 04.07.2020 Copyright $\subset 2020$ Heart, Vessels and Transplantation 
T wave peak to end (Tp-e) interval is recent marker for ventricular susceptibility for arrhythmias and repolarization heterogeneity (8-10). A prolonged Tp-e interval represents a period of susceptibility to ventricular arrhythmias. It was shown that this interval has been associated with increased risk of mortality in various congenital and structural heart diseases (11-15).

There is little information in current literature concerning ventricular arrhythmia susceptibility in ASD.

Our goal is to assess if there is an impact of shunt ratio on transmural repolarization in patients with ASD by using Tp-e interval, Tp-e/QT ratio, and Tpe/QTc ratio.

\section{Methods}

\section{Study participants}

Virtual data management system of Samsun Training and Research Hospital was retrospectively analyzed. One hundred and thirty-three patients who had been diagnosed with secundum ASD at outpatient clinic were enrolled. Their electrocardiograms were acquired and scanned. Echocardiographic calculations such as shunt ratio, pulmonary artery pressure and other measurements of right ventricle diameters were gained from previous reports in digital archive. Patients with significant coronary artery lesion, critically ill ASD patients (requiring pulmonary arterial hypertension-specific therapy), moderate or more severe degree valve disease other than tricuspid valve, left ventricular failure, any ASD type other than secundum ASD, left ventricular hypertrophy, atrial fibrillation, right or left bundle block or patients with implanted pacemaker or cardioverter/defibrillator were excluded. Informed consent was obtained from all patients before procedures. Study protocol was endorsed by local scientific board.

\section{Availability of Data and Materials}

Supplementary material consisting of supportive data is available from the corresponding author, (OCY), upon reasonable request.

\section{Measurement of Tp-e, QT and QRS Intervals from the 12-Lead ECG}

All ECGs were scanned. Utilization of a manual measuring tool and a magnifier for interval measurements; could be either inaccurate or time consuming. ECG papers were scanned and that allowed researcher to make measurements in virtual environment. All intervals were measured by blinded colleague with a virtual caliper on computer interface. Measurements of Tp-e interval were performed from precordial lead V5 as it was described (Fig. 1) (16). From the peak (or the nadir if it was negative) of $T$ wave to the end point where it reaches isoelectric line was measured for Tp-e interval.

The QT interval was measured from the beginning of the QRS complex to where T waves descend onto the isoelectric baseline. When an $U$ wave interrupted the $T$ wave before returning to isoelectric line, the QT interval was measured to the nadir of the bent segment between the T and $U$ waves. The QTc interval was calculated using the Bazett formula: QTc $(\mathrm{ms})=\mathrm{QT}$ measured/VRR (sec). QT interval was measured from precordial V5 lead.

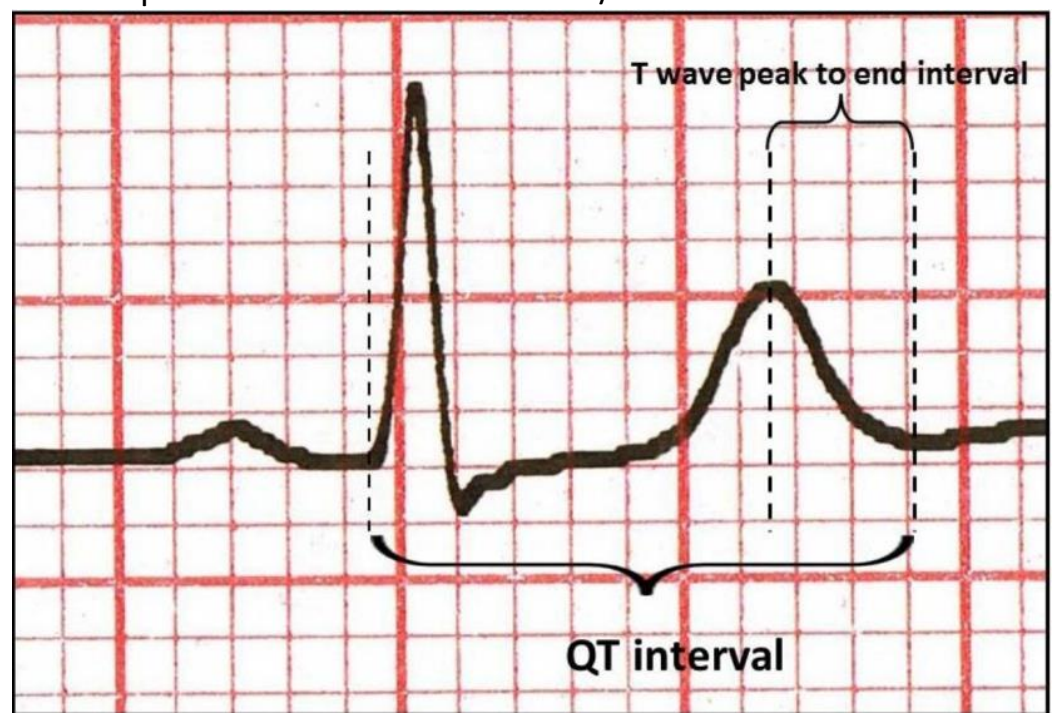

Figure 1. Measurement of intervals on electrocardiogram 


\section{Echocardiography}

Atrial septal defect shunt ratio was calculated noninvasively by echocardiography (17). Pulmonary perfusion ( $Q p)$ was calculated by multiplying right ventricle outflow tract (RVOT) time-velocity integral (in centimeters) and pi number and square of the half of the RVOT diameter (in millimeters). Systemic perfusion (Qs) was calculated by multiplying left ventricular outflow tract time-velocity integral (in centimeters) and pi number and square of the half of the left ventricular outflow tract diameter (in millimeters). Then $Q p$ was divided by Qs to calculate shunt ratio.

Also right ventricular midline/baseline/ end-diastolic base-to-apex distance, end-diastolic and end-systolic volumes, systolic pulmonary artery pressure (SPAP), left ventricular ejection fraction and tricuspid annular plane systolic excursion (TAPSE) were determined. Each echocardiogram was evaluated by 2 blinded sonographers.

\section{Statistical analysis}

All analyses were performed using SPSS $V 22.0$ for Windows (SPSS Inc., Chicago, Illinois, USA). We calculated the minimum number of the individuals that should be sampled with $90 \%$ power and 0.05 Type-I error as at least 58 ( $R$ 3.0.1. open source program). For parametric and continuous variables, independent-samples $\mathrm{t}$ test; for nonparametric and categorical variables, Chi-square test was used for univariate case-control comparisons for all cases vs. controls, respectively. A $P$ value of $<0.05$ was considered as significant. Spearman correlation coefficient was used for correlation analysis. Association of different variables with electrocardiographic and echocardiographic parameters and shunt ratio were separately calculated in univariate analysis.

\section{Results}

\section{Baseline data}

A total of 133 patients were grouped into two: 65 patients constituted low shunt ratio group $(<2.0)$, remaining 68 patients formed the group with high shunt ratio $(\geq 2.0)$. Mean age for patients with high shunt ratio was 40.5 (12.5) and for lower shunt group was $38.9(12.7)(p=0.463)$. High shunt group consisted of 47 female patients $(72.3 \%)$ whereas there were 44 female patients $(64.7 \%)$ in low shunt group $(p=0.358)$. Twenty three patients (35.4\%) of low shunt group had diabetes whereas there were 14 (20.6\%) patients with diabetes in high shunt group ( $p=0.834)$. High and low shunt groups did not differ in entity of hypertensive individuals (7 vs. 10 ) and smokers (31 vs. 32 ).

\section{ECG and echocardiographic variables}

Echocardiographic and ECG measurements are listed in Table 1. High shunt group had significantly longer Tp-e, QT and QTc intervals and higher Tp-e/QT and Tp-e/QTc ratios than low shunt group $(p<0.001$ for all).

Echocardiographic measurements of RV diameters (end-diastolic annulus line diameter, end-diastolic mid-line diameter, end-diastolic base-to-apex distance), RV volumes and SPAP were higher $(p<0.001)$, while TAPSE was significantly lower $(p<0.001)$ in high shunt group as compared to low shunt group. Mean left ventricular ejection fraction was similar in both high and low shunt groups ( $p>0.05)$.

\section{Correlations between parameters of transmural repolarization with ECG and echocardiographic variables}

Of all ECG parameters; Tp-e $(r=0.631, p<0.001), T p$ e/QT ( $r=0.531, \quad p<0.001)$ and Tp-e/QTc $(r=0.614$, $p<0.001)$ had moderate correlation with shunt ratio (Table 2). On the other hand, right ventricular enddiastolic $(r=0.783, p<0.001)$ and end-systolic volumes $(r=0.833, p<0.001)$, TAPSE $(r=-0.638, p<0.001)$ and SPAP $(0.677, p<0.001)$ showed strong correlation with shunt ratio (Table 3 ).

\section{Discussion}

Our results demonstrated that ASD patients whose shunt ratio are $\geq 2.0$ had longer Tp-e intervals and higher Tp-e/QT and Tp-e/QTc ratios than individuals with a lower shunt ratio and there is a positive correlation between shunt ratio and Tp-e interval, Tpe/QT and Tp-e/QTc ratios

Atrial septal defect is one of the most common congenital heart diseases. Frequency of atrial tachyarrhythmias and conduction diseases is very well studied in this population (18-20). Atrial arrhythmia prevalence shows correlation with size of shunt and occurrence of hemodynamic complications such as pulmonary artery hypertension, as well as with other comorbidities. However, there is a lack of scientific data regarding occurrence of ventricular arrhythmogenesis in this group of patients. Eryu et al. (21) investigated the RR and QT interval variability in a 
Table 1. Comparison of echocardiographic and electrocardiographic parameters between both groups

\begin{tabular}{|c|c|c|c|}
\hline Parameters & $\begin{array}{c}\text { Shunt ratio } \geq 2.0 \\
(n=68)\end{array}$ & $\begin{array}{c}\text { Shunt ratio }<2.0 \\
(n=65)\end{array}$ & $\mathbf{p}$ \\
\hline Mean shunt ratio & $2.2(0.1)$ & $1.3(0.2)$ & $<0.001$ \\
\hline $\mathrm{RV} 1, \mathrm{~mm}$ & $35.5(2.4)$ & $32.4(1.7)$ & $<0.001$ \\
\hline $\mathrm{RV} 2, \mathrm{~mm}$ & $28.0(2.2)$ & $24.7(1.4)$ & $<0.001$ \\
\hline RV3, mm & $66.5(2.8)$ & $64.0(1.9)$ & $<0.001$ \\
\hline RVEDV, ml & $185.4(13.9)$ & $147.62(7.0)$ & $<0.001$ \\
\hline RVESV, ml & $88.46(7.5)$ & $51.8(5.4)$ & $<0.001$ \\
\hline SPAP, mmHg & $35.5(7.6)$ & $19.3(5.2)$ & $<0.001$ \\
\hline TAPSE, mm & $11.2(2.9)$ & $16.2(1.6)$ & $<0.001$ \\
\hline LVEF, \% & $60.9(3.7)$ & $61.0(4.0)$ & 0.859 \\
\hline QT, msec & $409.9(62.7)$ & $361.4(35.7)$ & $<0.001$ \\
\hline QTc, msec & $459.7(44.0)$ & $428.2(43.1)$ & $<0.001$ \\
\hline Tp-e, msec & $103.0(22.1)$ & $76.2(10.2)$ & $<0.001$ \\
\hline Tp-e/QT ratio & $0.25(0.03)$ & $0.21(0.02)$ & $<0.001$ \\
\hline Tp-e/QTc ratio & $0.22(0.03)$ & $0.17(0.02)$ & $<0.001$ \\
\hline \multicolumn{4}{|c|}{$\begin{array}{l}\text { Data are presented as mean (SD) } \\
\text { LVEF - left ventricular ejection fraction, ml - milliliter, mm - millimeter, msec - millisecond, QTc - corrected } \\
\text { QT, RV1 - right ventricular end-diastolic annulus line diameter, RV2 - right ventricular end-diastolic mid- } \\
\text { line diameter, RV3 - right ventricular end-diastolic base-to-apex distance, RV EDV - right ventricular end- } \\
\text { diastolic volume, RVESV - right ventricular end-systolic volume, SPAP - systolic pulmonary artery pressure, } \\
\text { TAPSE - tricuspid annular plane systolic excursion, Tp-e- T wave peak to end interval }\end{array}$} \\
\hline
\end{tabular}




\begin{tabular}{|l|c|c|}
\hline \multirow{2}{*}{ Table 2. Correlations between electrocardiographic parameters and shunt ratio } \\
\hline \multirow{2}{*}{ Qariables $\mathrm{msec}$} & $\mathbf{2}$ & $\mathbf{p}$ \\
\cline { 2 - 3 } & 0.457 & $<0.001$ \\
\hline QTc, msec & 0.375 & $<0.001$ \\
\hline Tpe, msec & 0.631 & $<0.001$ \\
\hline Tpe/QT & 0.531 & $<0.001$ \\
\hline Tpe/QTc & 0.614 & $<0.001$ \\
\hline Tp-e - T wave peak to end interval, msec - millisecond, QTc - corrected QT \\
\hline
\end{tabular}

\begin{tabular}{|l|c|c|}
\hline \multirow{2}{*}{ Table 3. Correlations between echocardiographic parameters and shunt ratio } \\
\hline \multirow{2}{*}{ RV1 } & $\mathbf{2}$ & $\mathbf{p}$ \\
\cline { 2 - 3 } & 0.553 & $<0.001$ \\
\hline RV2 & 0.569 & $<0.001$ \\
\hline RV3 & 0.393 & $<0.001$ \\
\hline RVESV & 0.783 & $<0.001$ \\
\hline LVEF & 0.833 & $<0.001$ \\
\hline SPAP & 0.008 & 0.926 \\
\hline TAPSE & 0.677 & $<0.001$ \\
\hline $\begin{array}{l}\text { LVEF - left ventricular ejection fraction, ml - milliliter, mm - millimeter, msec - millisecond, RV1 - } \\
\text { right ventricular end-diastolic annulus line diameter, RV2 - right ventricular end-diastolic mid-line } \\
\text { diameter, RV3 - right ventricular end-diastolic base-to-apex distance, RVEDV - right ventricular } \\
\text { end- diastolic volume, RVESV - right ventricular end systolic volume, SPAP - systolic pulmonary } \\
\text { artery pressure, TAPSE - tricuspid annular plane systolic excursion }\end{array}$ \\
\hline
\end{tabular}


Authors came to a conclusion that these repolarization indices provided information on alteration of sinus node autonomic control and the pathophysiology of myocardial repolarization. A more recent study (22) was able to show prolonged QT intervals in children with relevant hemodynamic impairment. Authors reach to a conclusion that after closure operation patients' QTc interval shortened significantly. This finding may create a discussion point for our article's intellectual basis. In our study, we utilized another myocardial repolarization index, the Tp-e interval, in concordance with QT interval, to determine ventricular arrhythmia susceptibility in a group of ASD patients with relatively lower shunt ratio.

Miscellaneous methods were utilized in order to assess myocardial repolarization including QT dispersion (QTd), corrected QT dispersion (cQTd), and transmural dispersion of repolarization. Recent studies demonstrated that Tp-e interval can be used as an index of total (transmural, apico-basal, and global) repolarization disparity $(23,24)$. Increased Tpe interval might be a useful index to predict ventricular tachyarrhythmias and cardiovascular mortality (25). Furthermore, Tp-e/QT ratio has been suggested to be a more accurate measure for the dispersion of ventricular repolarization compared to QTd, cQTd, and Tp-e intervals, which is independent of alterations in heart rate (26). Also, these markers may be used as an ECG indexes of ventricular arrhythmogenesis and sudden cardiac death $(16,20)$. Previous studies showed that prolongation of Tp-e interval was associated with increased mortality in various clinical cardiac conditions $(11,12,14,16)$. The novel repolarization indexes Tp-e interval and Tp-e/QT ratio, has not been studied in ASD patients before.

Abnormal hemodynamics in ASD patients may play the most important role in ventricular arrhythmogenesis. Left-to-right shunt increases right atrial and eventually right ventricular volume and pressure load to some extent (1). Abnormal right ventricle workload may promote fibrosis in myocardial tissue that would precede an arrhythmic risk. On the other hand, right ventricular volume and pressure overload shift the interventricular septum towards left ventricle. This displacement precedes an impaired diastolic filling and a reduced stroke volume. This would also provoke fibrosis in ventricle. Our patients with larger shunts had their right ventricular end-systolic and end-diastolic volumes significantly increased, which clearly indicates right heart failure. Another theory for conduction heterogeneity in these patients is that, left ventricular diastolic impairment might lead to autonomic imbalance (27) which would directly affect Tp-e and related intervals. Eryu et al (21) asserted that there was an increase in sympathetic control of the heart or a decrease in parasympathetic control of the heart in children with ASD. In our opinion there should be other factors in charge which involve microarchitecture and ion channels and signal transmission than macro changes in heart chambers.

\section{Study limitations}

The most important restriction of our study is the limited number of patients. Another limitation we did not assess the association between ventricular arrhythmias with Tp-e interval and Tp-e/QT ratio. Also study population could not be followed-up prospectively for ventricular arrhythmic episodes. Large-scale prospective studies are needed to determine the predictive value of prolonged Tp-e interval and increased Tp-e/QT ratio in this population.

\section{Conclusion}

Our study revealed that transmural repolarization is more deteriorated in ASD patients with large shunts and dilated right ventricle than individuals with a lower shunt ratio. Tp-e interval and Tp-e/QT ratio might be a useful marker of arrhythmia susceptibility in this group.

Peer-review: External and internal Conflict of interest: None to declare Authorship: O.C.Y Acknowledgments and funding: None to declare

\section{References}

1. Van der Linde D, Konings EEM, Slager MA, Witsenburg M, Helbing WA, Takkenberg JJ, et al. Birth prevalence of congenital heart disease worldwide: a systematic review and meta-analysis. J Am Coll Cardiol 2011; 58:2241-7.

2. Kuijpers JM, Mulder BJ, Bouma BJ. Secundum atrial septal defect in adults: a practical review and recent developments. Neth Heart J 2015; 23:205-11.

3. Moons $P$, Bovijn L, Budts $W$, Belmans $A$, Gewillig M. Temporal trends in survival to adulthood among patients born with congenital heart disease from 1970 to 1992 in Belgium. Circulation 2010; 122: 2264-72. 
4.Yong G, Khairy P, Guise PD, Dore A, Marcotte F, Mercier LA, et al. Pulmonary arterial hypertension in patients with transcatheter closure of secundum atrial septal defects a longitudinal study. Circ Cardiovasc Interv 2009; 2:455-62.

5.Geva T, Martins JD, Wald RM. Atrial septal defects. Lancet 2014; 383: 1921-32.

6. Chubb H, Whitaker J, Williams SE, Head CE, Chung NA, Wright MJ, et al. Pathophysiology and management of arrhythmias associated with atrial septal defect and patent foramen ovale. Arrhythm Electrophysiol Rev 2014; 3: 168-72.

7. Morton JB, Sanders P, Vohra JK, Sparks PB, Morgan $J G$, Spence SJ, et al. Effect of chronic right atrial stretch on atrial electrical remodeling in patients with an atrial septal defect. Circulation 2003; 8: 1775-82.

8. Taggart $P$, Sutton $P M$, Opthof $T$, Coronel R, Trimlett $\mathrm{R}$, Pugsley $\mathrm{W}$, et al. Transmural repolarization in the left ventricle in humans during normoxia and ischaemia. Cardiovasc Res 2001; 50: 454 - 62.

9. Opthof $T$, Coronel $R$, Janse MJ. Is there a significant transmural gradient in repolarization time in the intact heart?: Repolarization gradients in the intact heart. Circ Arrhythm Electrophysiol. 2009; 2: 89-96.

10. Antzelevitch C, Sicouri S, Litovsky SH, Lukas A, Krishnan SC, Di Diego JM, et al. Heterogeneity within the ventricular wall. Electrophysiology and pharmacology of epicardial, endocardial, and M cells. Circ Res 1991; 69: 1427-9.

11.Topilski I, Rogowski O, Rosso R, Justo D, Copperman Y, Glikson $\mathrm{M}$ et al. The morphology of the QT interval predicts torsade de pointes during acquired bradyarrhythmias. J Am Coll Cardiol 2007; 49: 320-8.

12. Shimizu M, Ino H, Okeie K, Yamaguchi M, Nagata $M$, Hayashi $K$, et al. T-peak to T-end interval may be a better predictor of high-risk patients with hypertrophic cardiomyopathy associated with a cardiac troponin I mutation than QT dispersion. Clin Cardiol 2002; 25: 335-9.

13. Yontar OC, Karaagac K, Tenekecioglu E, Tutuncu A, Demir $M$, Melek M. Assessment of ventricular repolarization inhomogeneity in patients with mitral valve prolapse: value of T wave peak to end interval. Int J Clin Exp Med 2014; 7: 2173-8.

14. Haarmark C, Hansen PR, Vedel-Larsen E, Pedersen $\mathrm{SH}$, Graff C, Andersen MP, et al. The prognostic value of the Tpeak-Tend interval in patients undergoing primary percutaneous coronary intervention for STsegment elevation myocardial infarction. J Electrocardiol 2009; 42: 555-60.

15. Karaagac K, Yontar OC, Tenekecioglu E, Vatansever F, Ozluk OA, Tutuncu A, et al. Evaluation of Tp-Te interval and Tp-Te/QTc ratio in patients with coronary artery ectasia. Int J Clin Exp Med 2014; 7: 2865-70.

16. Castro Hevia J, Antzelevitch C, Tornés Bárzaga F, Dorantes Sánchez M, Dorticós Balea F, Zayas Molina $\mathrm{R}$, et al. Tpeak-Tend and Tpeak-Tend dispersion as risk factors for ventricular tachycardia/ventricular fibrillation in patients with the Brugada syndrome. J Am Coll Cardiol 2006; 47: 1828-34.

17. Henry WL, DeMaria A, Gramiak R, King DL, Kissio JA, Popp RL, et al. Report of the American Society of Echocardiography Committee on Nomenclature and Standards in two-dimensional imaging. Circulation 1980; 62: 212-7.

18. Chubb H, Whitaker J, Williams SE, Head CE, Chung NA, Wright MJ, et al. Pathophysiology and management of arrhythmias associated with atrial septal defect and patent foramen ovale. Arrhythm Electrophysiol Rev 2014; 3: 168-72.

19. Vecht JA, Saso S, Rao C, Dimopoulos K, Grapsa J, Terracciano $\mathrm{CM}$, et al. Atrial septal defect closure is associated with a reduced prevalence of atrial tachyarrhythmia in the short to medium term: a systematic review and meta-analysis. Heart 2010; 96: 1789-97.

20. Brouwer C, Hazekamp MG, Zeppenfeld K. Anatomical substrates and ablation of reentrant atrial and ventricular tachycardias in repaired congenital heart disease. Arrhythm Electrophysiol Rev 2016; 5: 150-60.

21. Eryu $Y$, Hata $T$, Nagatani A, Funamoto $Y$, Uchida $H$, Fujino $M$, et al. Electrocardiographic $R R$ and $Q T$ interval variability in patients with atrial septal defect and healthy children. Pediatr Cardiol 2017; 38: 582-7.

22. Rucklova K, Koubsky K, Tomek V, Kubus P, Janousek J. Prolonged repolarization in atrial septal defect: An example of mechanoelectrical feedback due to right ventricular volume overload. Heart Rhythm 2016; 13: 1303-8.

23. Kors JA, Ritsema van Eck HJ, van Herpen G. The meaning of the Tp-Te interval and its diagnostic value. J Electrocardiol 2008; 41: 575-80.

24. Antzelevitch C, Sicouri S, Di Diego JM, Burashnikov A, Viskin S, Shimizu W. et al. Does Tpeak-Tend provide an index of transmural dispersion of repolarization? Heart Rhythm 2007; 4: 1114-6.

25. Smetana P, Schmidt A, Zabel M, Hnatkova K, Franz $M$, Huber $K$, et al. Assessment of repolarization heterogeneity for prediction of mortality in cardiovascular disease: peak to the end of the T wave interval and nondipolar repolarization components. J Electrocardiol 2011; 44: 301-8. 
Heart, Vessels and Transplantation 2020; 4: doi: 10.24969/hvt.2020.206

26. Gupta P, Patel C, Patel H, Narayanaswamy S, Malhotra B, Green JT, et al. T(p-e)/QT ratio as an index of arrhythmogenesis. J Electrocardiol 2008; 41: 56774.
27. Massin M, von Bernuth G. Clinical and haemodynamic correlates of heart rate variability in children with congenital heart disease. Eur J Pediatr 1998; 157: 967-1.
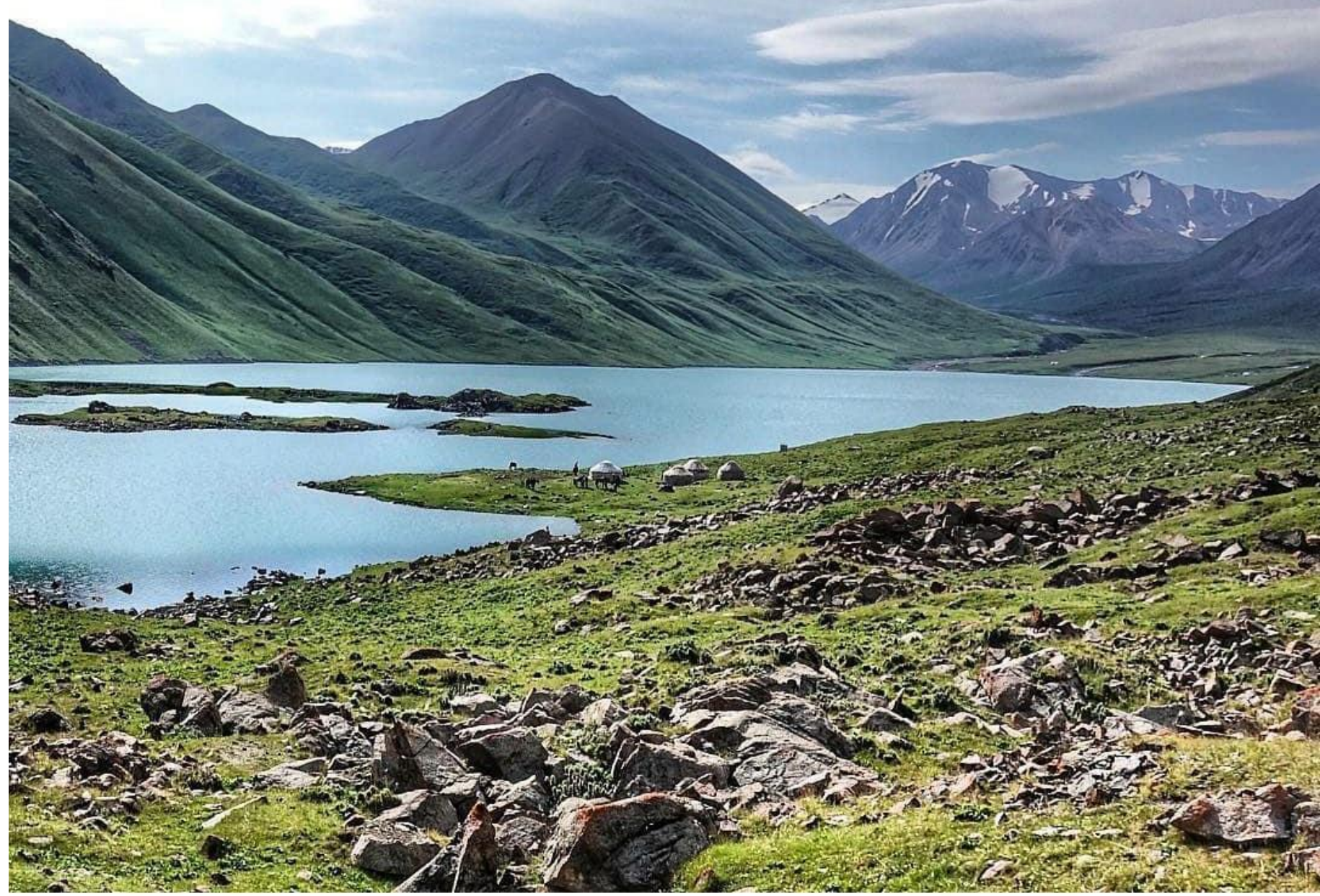

The lake in high-altitude Kochkor region, Naryn, Kyrgyzstan. Ulan Tursunbekov, Bishkek, Kyrgyzstan. 
